\title{
Enhancing Color Purity and Stable Efficiency of White Organic Light Diodes by Using Hole-Blocking Layer
}

\author{
Chien-Jung Huang, ${ }^{1}$ Kan-Lin Chen, ${ }^{2}$ Dei-Wei Chou, ${ }^{3}$ Yu-Chen Lee, ${ }^{1}$ and Chih-Chieh Kang ${ }^{4}$ \\ ${ }^{1}$ Department of Applied Physics, National University of Kaohsiung, Nanzih, Kaohsiung 81148, Taiwan \\ ${ }^{2}$ Department of Electronic Engineering, Fortune Institute of Technology, Kaohsiung 83160, Taiwan \\ ${ }^{3}$ Department of Aviation \& Communication Electronics, Air Force Institute of Technology, Kaohsiung 82044, Taiwan \\ ${ }^{4}$ Department of Electro-Optical Engineering, Southern Taiwan University of Science and Technology, Tainan 71005, Taiwan
}

Correspondence should be addressed to Chien-Jung Huang; chien@nuk.edu.tw and Kan-Lin Chen; klchen@fotech.edu.tw

Received 18 May 2014; Accepted 4 June 2014; Published 17 June 2014

Academic Editor: Fu-Ken Liu

Copyright (C) 2014 Chien-Jung Huang et al. This is an open access article distributed under the Creative Commons Attribution License, which permits unrestricted use, distribution, and reproduction in any medium, provided the original work is properly cited.

\begin{abstract}
The organic light-emitting diodes with triple hole-blocking layer (THBL) formation sandwich structure which generate white emission were fabricated. The 5,6,11,12-tetraphenylnapthacene (Rubrene), (4,4'-N, $\mathrm{N}^{\prime}$-dicarbazole)biphenyl (CBP), and 4,4'bis (2,2' diphenylvinil)-1,1'-biphenyl (DPVBi) were used as emitting materials in the device. The function of CBP layer is not only an emitting layer but also a hole-blocking layer (HBL), and the Rubrene was doped into the CBP. The optimal configuration structure was indium tin oxide (ITO)/Molybdenum trioxide $\left(\mathrm{MoO}_{3}\right)(5 \mathrm{~nm}) /[4,4$-bis[N-(1-naphthyl)-N-phenylamino]biphenyl (NPB) $(35 \mathrm{~nm}) / \mathrm{CBP}$ (HBL1) $(5 \mathrm{~nm}) / \mathrm{DPVBi}$ (I) $(10 \mathrm{~nm}) / \mathrm{CBP}$ (HBL2) : Rubrene $(4: 1)(3 \mathrm{~nm}) / \mathrm{DPVBi}$ (II) (30 nm)/CBP (HBL3) $(2 \mathrm{~nm}) / 4,7$-diphenyl-1,10-phenanthroline (BPhen) $(10 \mathrm{~nm}) /$ Lithium fluoride $(\mathrm{LiF}) /$ aluminum $(\mathrm{Al})$. The result showed that the device with Rubrene doped in CBP (HBL2) exhibited a stable white emission with the color coordinates of $(0.322,0.368)$, and the coordinate with the slight shift of $\pm \Delta_{x, y}=(0.001,0.011)$ for applied voltage of $8-12 \mathrm{~V}$ was observed.
\end{abstract}

\section{Introduction}

The white organic light-emitting diodes (WOLED) have attracted considerable attention in recent years due to their widely used applications such as full-color displays and solid-state lighting sources [1-3]. Many approaches have been tried to realize full-color displays, and it requires three basic emitting colors, red, green, and blue [4-6]. The white emission is usually obtained by using doping method, which is red, green, and blue (RGB), or the doping of two complementary colors (blue and yellow) into respective host layer [7-17]. In recent years, the 5,6,11,12-tetraphenylnapthacene (Rubrene) is widely used as yellow material, which has better light emitting efficiency, high color saturation, and strong charge carriers capture efficiency. In addition, the photoluminescence (PL) quantum efficiency of Rubrene can be near $100 \%$ [18-21]. Wang et al. utilized the structure of [4,4-bis[N-(1-naphthyl)- $\mathrm{N}$ phenylamino]biphenyl (NPB)/4,4' - Bis $(9 \mathrm{H}$-carbazol-9-yl) biphenyl (CBP): Rubrene/CBP: N-(4-((E)-2-(6-((E)-4(diphenylamino)styryl)naphthalen-2-yl)vinyl)phenyl)-Nphenylbenzenamine (N-BDAVBi) to make double yellow and blue emitting layer, resulting in Commission International de L'Eclairage (CIE) coordinates from $(0.27,0.37)$ to $(0.28$, 0.36 ) at applied voltage from $6 \mathrm{~V}$ to $16 \mathrm{~V}$ [4]. Xue et al. used the structure of $4,4^{\prime}$-bis $\left(2,2^{\prime}\right.$ diphenylvinil)-1,1'-biphenyl (DPVBi)/CBP/CBP: fac-tris(2-phenylpyridine)iridium (Ir (ppy)3): Rubrene/ and introduced a bipolar transport CBP layer between fluorescent and the phosphor-sensitizedfluorescent layer, resulting in the CIE coordinates of $(0.22$, 0.33 ) [22]. Among various configurations for WOLED, the multiple hole-blocking layers (HBL) structure has been proved to be an effective approach for high performance of device, by confining charge carriers and exciton within the multiple emitting layers (EML); thus the charge carrier 

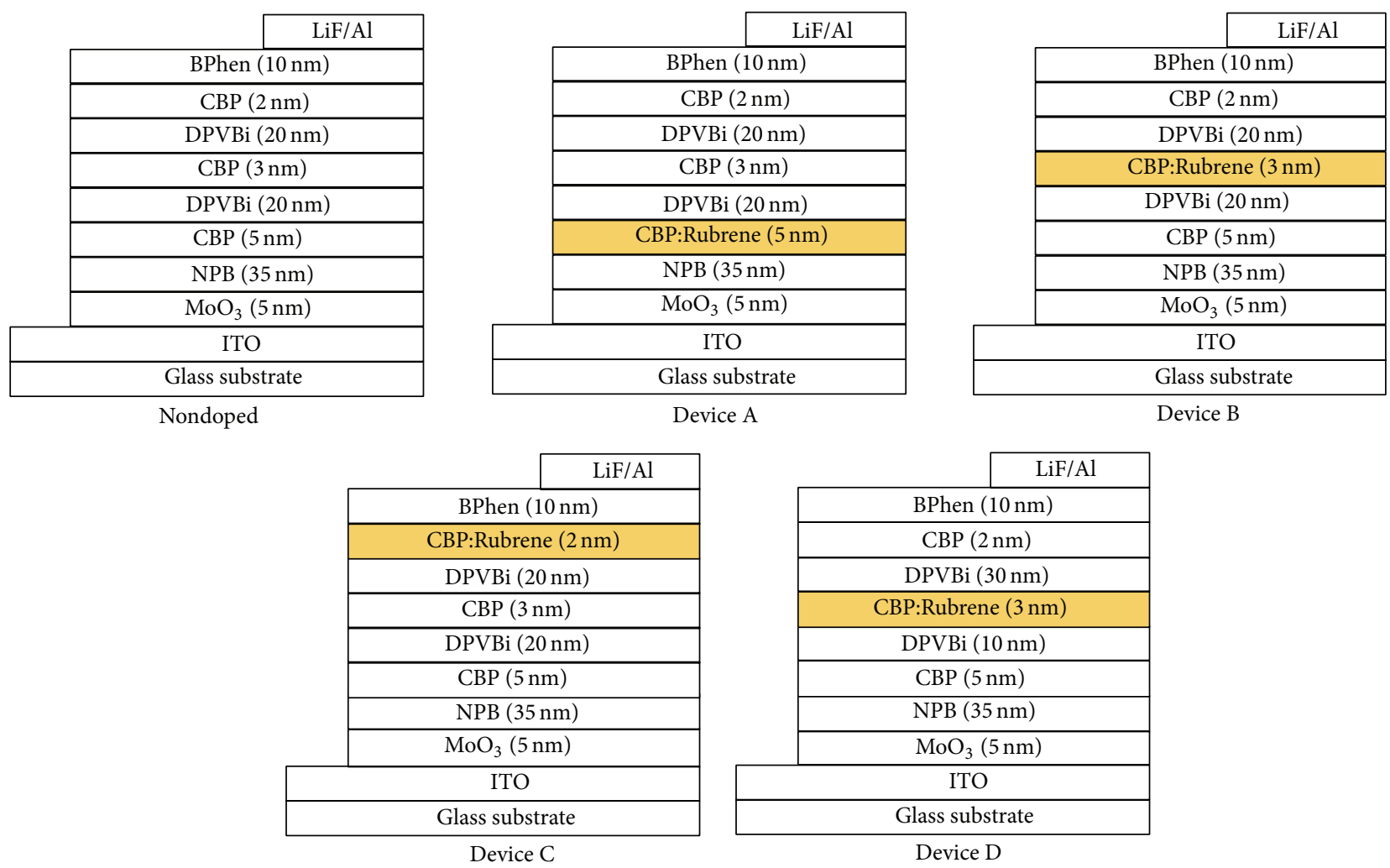

FIGURE 1: The schematic structures of the devices A-D.

recombination efficiency and exciton formation can be enhanced [23-27].

In this study, the WOLED was obtained by using structure of triple-hole blocking layer (THBL), which consisted of three $\mathrm{CBP}$ layers. It has excellent electrons transport and holes blocking ability. The CBP layer doped with Rubrene can effectively control the carrier recombination in the emitting layer that will favor the white light emission. It is due to the fact that the highest occupied molecular orbital (HOMO) of CBP is higher than that of Rubrene. However, we used the THBL structure so that the carrier can be effectively confined and controlled at the interface of CBP/DPVBi, resulting that the CIE color coordinates of devices can be close to the standard CIE of white light emission. Simultaneously, the mechanism of devices with different Rubrene doped CBP layers is presented.

\section{Experimental Part}

The indium tin oxide (ITO) coated glass with a sheet resistance of approximately $15 \Omega /$ sq was consecutively cleaned in ultrasonic bath containing detergent water, acetone, ethanol, and deionized (DI) water for $20 \mathrm{~min}$ each and then dried with a nitrogen $\left(\mathrm{N}_{2}\right)$ flow. The organic materials used in the study are NPB (99\%, E-Ray), CBP (97\%, Aldrich), DPVBi (99.9\%, Lumintech), 4,7-diphenyl-1,10phenanthroline (BPhen) (99\%, E-Ray), and lithium fluoride (LiF) ( $\geqq 99 \%$, Aldrich), which were without further sublimation. All of the deposition procedures involved thermal evaporation in a vacuum chamber $\left(<10^{-6}\right.$ Torr $)$. The organic layers were sequentially evaporated onto the ITO coated-glass substrate. The deposition rates of all organic materials were monitored by an oscillating quartz thickness monitor (Sigma, SID-142). The deposition rates were about $0.2 \sim 0.5 \AA / s$ for organic layers and $5 \AA /$ s for cathode aluminum (Al) and $\mathrm{LiF}$. The schematic structures of devices are shown in Figure 1. The CIE coordinates, electroluminescence (EL) spectra and luminance, were measured by the photospectrometer (Kollmorgen Instrument PR-655, USA) and the current-voltage characteristics were simultaneously measured by the programmable power source (Keithley SourceMeter 2400, USA), controlled using computer software (Chief I-V-L system, Taiwan). All measurements were carried out at room temperature under ambient.

\section{Results and Discussion}

The schematic energy band diagrams of devices are shown in Figure 2. In the device, the NPB is used as hole-injecting layer and hole-transport layer. The CBP layers are used as the EML and the HBLs. In Figure 2, HBL1, HBL2, and HBL3 represented the $\mathrm{HBL}$ that was close to the anode side, in the middle, and close to cathode, respectively, which forms the so-called THBL that can control the carrier recombination in the EML to adjust the emission color of devices. The Rubrene is used as a guest doping material to emit the yellow light for achieving the white light emission, and the DPVBi is used as blue-emitting layer. The Rubrene is doped into the 


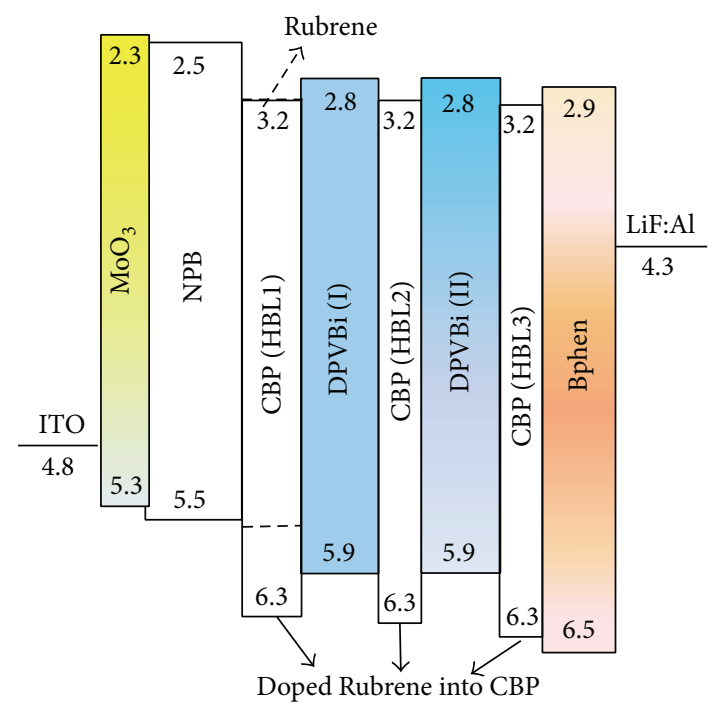

FIgURE 2: The schematic energy band diagrams of devices.

CBP layer rather than the DPVBi layer. This is due to the fact that CBP has higher carrier trapping ability than that of DPVBi. Nevertheless, if the Rubrene is doped into the DPVBi layer, it will cause the attenuation in luminous efficiency of DVPBi. Figure 3 shows the EL spectra of devices at current density of $100 \mathrm{~mA} / \mathrm{cm}^{2}$. The peak wavelengths of nondoped device are at $416 \mathrm{~nm}$ and $468 \mathrm{~nm}$ originating from CBP and DPVBi, respectively. It shows the main peak emission from CBP (HBL1) and minor peak emission from DPVBi (I), which implies that the recombination zone of excitons is at the range of CBP (HBL1) and DPVBi (I). To confirm the excitons recombination zone and to achieve white light emission, the Rubrene is doped into the CBP (HBL1) in device A. The emission peaks of device $A$ are at $460 \mathrm{~nm}$ and $564 \mathrm{~nm}$ wavelength from DPVBi (I) and Rubrene, respectively. It indicates that most excitons recombination happens within the DPVBi (I) layer and the CBP (HBL1) layer, resulting in the yellow light emission of Rubrene. In addition, the emission peak from CBP (HBL1) in device A has disappeared due to the energy transfer from host to guest, that is, CBP (HBL1) to Rubrene. Furthermore, a part of blue light emitted from DPVBi (I) is absorbed by Rubrene molecular, leading to the reduction in emission peak of DPVBi (I). However, the intensity of yellow light is higher than that of blue light. Hence, in order to reach the balance between intensity of yellow light and blue light, the doping position of Rubrene changed to the CBP (HBL2) of device B, which can prevent the blue light emitted from the DPVBi (I) layer from being absorbed by the Rubrene. As a result, the relative intensity of yellow to blue emission decreases in device B. It is also found that the intensity of blue emission is higher than that of yellow emission, indicating that most of excitons recombination is at the range of CBP (HBL1)/DPVBi (I) while few excitons trapping and formation is at CBP (HBL2) layer. In device $\mathrm{C}$, the Rubrene is doped into the CBP (HBL3), and the intensity of yellow light is almost negligible. It is attributed to the fact that the Rubrene doping layer is far away from the exciton recombination zone, which is consistent with previous discussion. The main excitons recombination zone of device is at the interface of CBP (HBL1)/DPVBi (I), and the intensity of blue emission in device $B$ is higher than that of yellow emission, so the thickness of the DPVBi (I) layer nearing the CBP (HBL1) should be decreased to balance the yellow and blue emission. This is the reason why the device $\mathrm{D}$ based on the structure of device $\mathrm{B}$ which is fabricated. In device $\mathrm{D}$, the thickness of DPVBi (I) layer decreased to $10 \mathrm{~nm}$ and the thickness of DPVBi (II) layer increased to $30 \mathrm{~nm}$. Compared with device $\mathrm{B}$, the yellow intensity in device $\mathrm{D}$ is enhanced while the blue intensity is decreased a little. This is due to the fact that the amount of exciton in CBP (HBL2) increases and the amount of exciton in DPVBi (I) layer reduces, resulting from a decrease in the thickness of DPVBi (I) layer. The intensity of yellow emission from Rubrene is almost the same with the intensity of blue emission from DPVBi in device D by adjusting the thickness of DPVBi layer based on the structure of device B. Figure 3(b) shows the CIE coordinates of various devices at current density of $100 \mathrm{~mA} / \mathrm{cm}^{2}$. The CIE coordinates of devices A-C are $(0.431$, $0.419),(0.258,0.331)$, and $(0.218,0.292)$, respectively. It can be seen that the CIE coordinates of devices A-C are far away from the standard CIE coordinate of white emission. The CIE coordinate of device D is $(0.322,0.368)$ and is close to the standard CIE coordinate of white emission.

Figure 4(a) shows the EL spectra of device D at applied voltage of 8-12 V. It can be seen that the EL spectra show 416, 468, and $560 \mathrm{~nm}$ wavelengths for CBP, DPVBi, and Rubrene, respectively. The ratio of blue and yellow emission intensity in EL spectra is almost the same at different applied voltages, indicating that the excitons recombination zone of device $\mathrm{D}$ is steady. In other words, the ratio is not changed as the applied voltage switches. Figure 4(b) shows CIE coordinates of device $\mathrm{D}$ at different applied voltages. The $\mathrm{CIE}$ coordinates of device D at $8-12 \mathrm{~V}$ are $(0.321,0.379),(0.324,0.393),(0.319$, $0.389),(0.315,0.379)$, and $(0.322,0.368)$, respectively. The difference in CIE coordinates of device $\mathrm{D}$ is $\pm \Delta_{x, y}=(0.001$, 0.011 ). The result shows that the excitons can be confined in the recombination zone by using THBL structure; that is, the holes are confined and accumulated in the HOMO level of CBP (HBL1)/DPVBi (I)/CBP (HBL2) potential well in device $\mathrm{D}$ due to the existence of potential barrier at the CBP (HBL1)/DPVBi (I) and DPVBi (I)/CBP (HBL2) heterointerfaces. As a result, the balance of electron and hole can be improved by using the THBL structure, and thus the CIE coordinates stability of device $\mathrm{D}$ is achieved.

The current density-voltage, luminance-voltage, and luminous efficiency-current density characteristics of devices were shown in Figure 5. At a current density of $10 \mathrm{~mA} / \mathrm{cm}^{2}$, the turn-on voltages are $6.9 \mathrm{~V}, 9 \mathrm{~V}, 11.2 \mathrm{~V}$, and $8.2 \mathrm{~V}$ for devices $\mathrm{A}-\mathrm{D}$, respectively. The turn-on voltage of device $\mathrm{A}$ is lower than that of other devices. It is attributed to the fact that the barrier of energy level between NPB and CBP is $0.8 \mathrm{eV}$ and is higher than that of the one between NPB and Rubrene, resulting that the hole in device A can easily inject into Rubrene of bipolar molecules in CBP (HBL1) due to a substantially higher carrier affinity [28]. Besides, it 


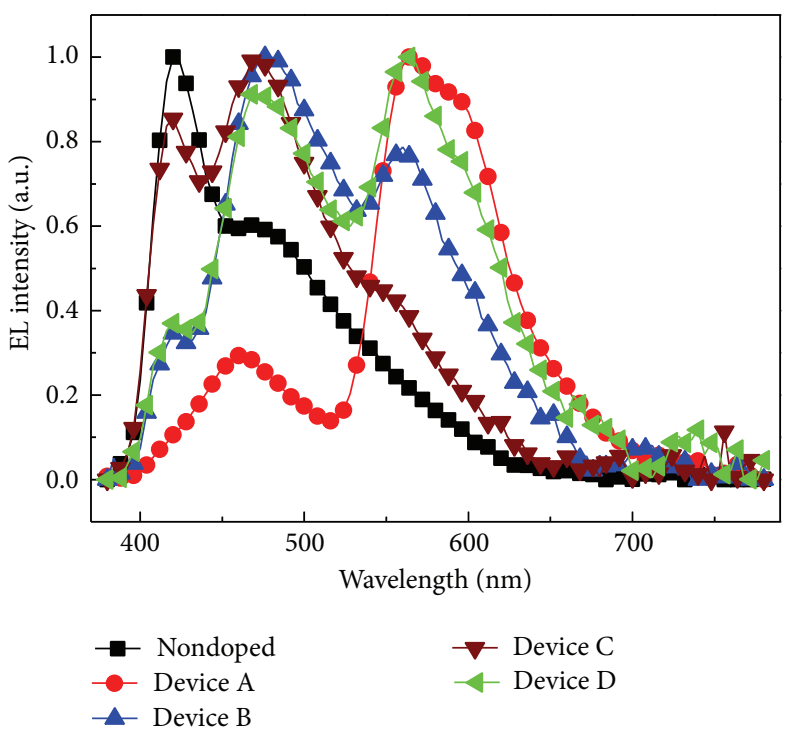

(a)

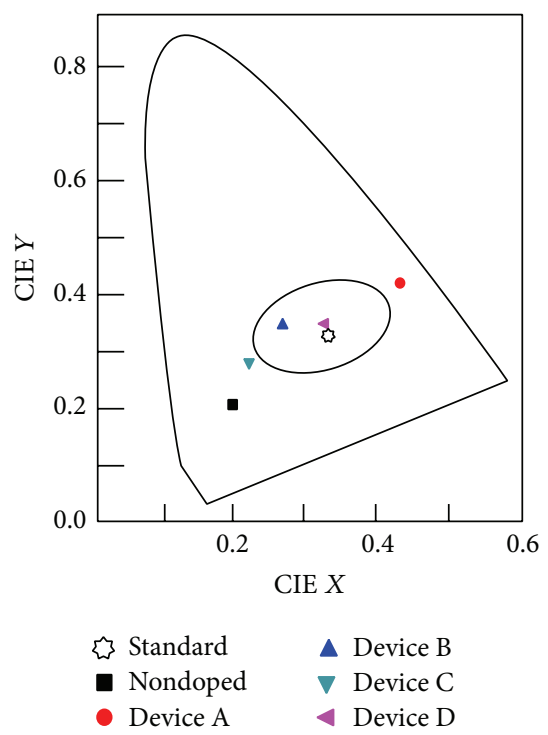

(b)

FIGURE 3: (a) EL spectra characteristics of various devices at injection current density of $100 \mathrm{~mA} / \mathrm{cm}^{2}$. (b) CIE coordinates of various devices at current density of $100 \mathrm{~mA} / \mathrm{cm}^{2}$.

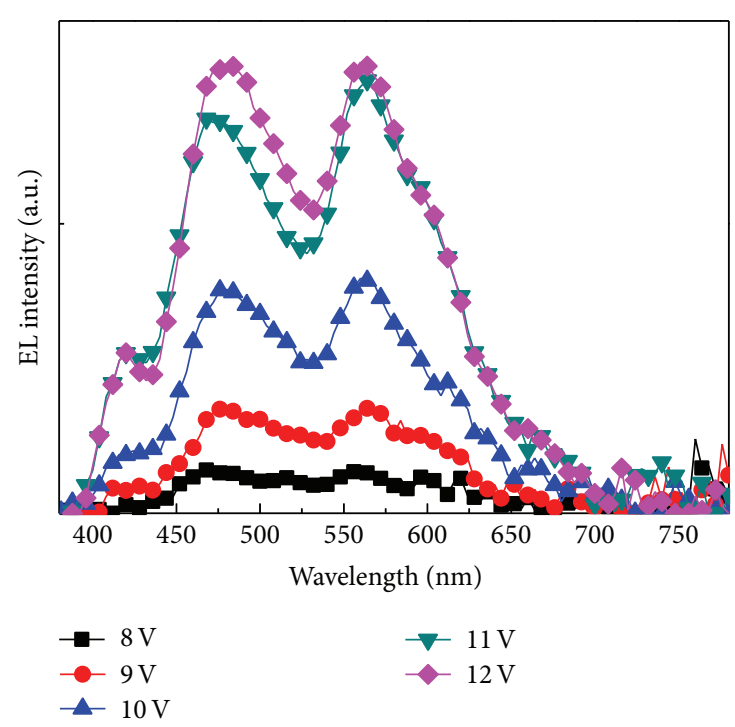

(a)
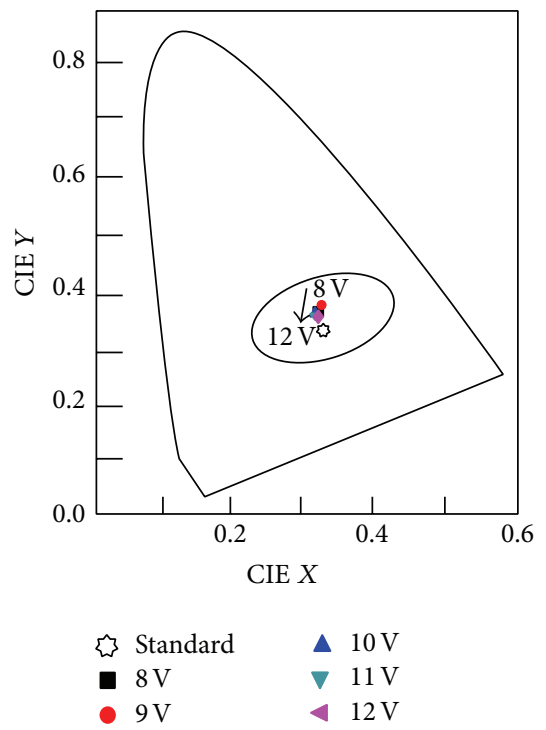

(b)

FIgURE 4: (a) The EL intensity spectra characteristics of the device D at applied voltage of 8-12 V. (b) The CIE coordinates of device D at applied voltage of $8-12 \mathrm{~V}$.

can be seen that the turn-on voltage of device $\mathrm{D}$ is lower than that of device $\mathrm{B}$. The phenomenon is attributed to the fact that the DPVBi (I) layer thickness of device D becomes thinner than that of device $B$, which enables Rubrene in CBP (HBL2) to be more close to the recombination zone of exciton. Furthermore, it is found that the efficiency of device A has a roll-off phenomenon (see Figure 5(c)). This is due to the fact that there is a peak wavelength of $460 \mathrm{~nm}$ in normalized EL spectra and it enhances with the increase at applied voltage of $7-10 \mathrm{~V}$, as shown in inset of Figure 5(c).
The minority hole blocked by barrier of NPB/CBP (HBL1) will cross the barrier of CBP (HBL1) and inject into DPVBi (I) with the increasing voltage, leading to the decrease of excitons at $\mathrm{CBP}$ (HBL1) and the reduction of energy transfer from CBP host to Rubrene guest, and thereby decrease efficiency of Rubrene. However, the whole efficiency of device A is reduced with the increasing current density. In contrast, the roll-off phenomenon is barely for device D. The maximum luminance and maximum luminous efficiency of the device $\mathrm{D}$ is $2410 \mathrm{~cd} / \mathrm{m}^{2}, 2.05 \mathrm{~cd} / \mathrm{A}$ at $12 \mathrm{~V}$, respectively. The result 


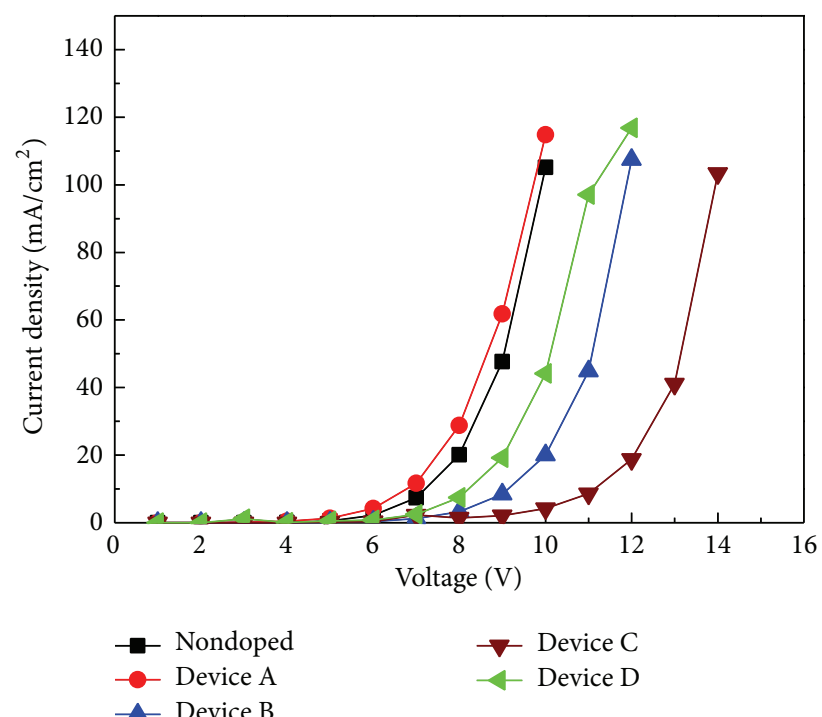

(a)

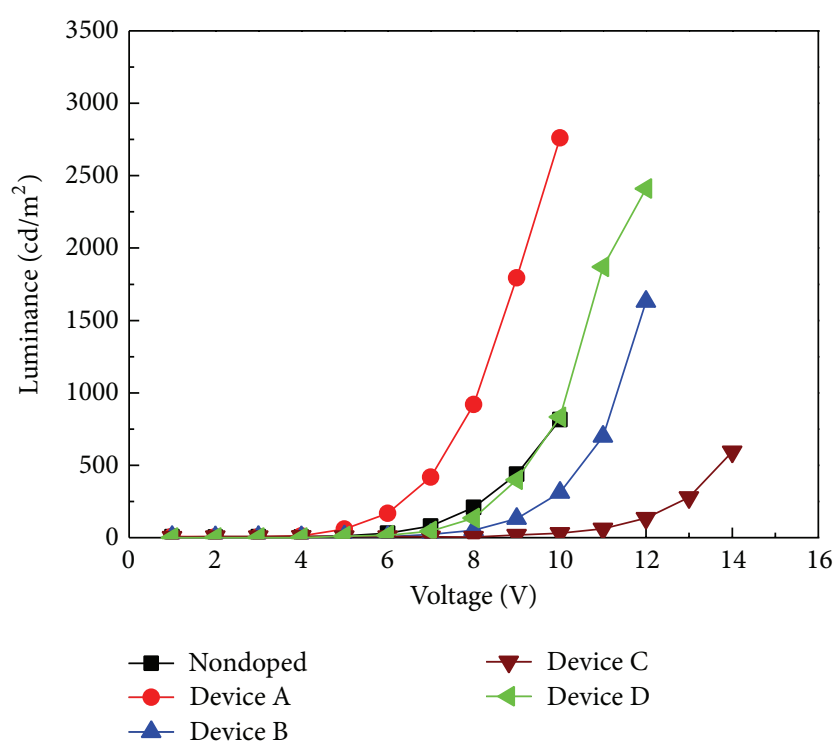

(b)

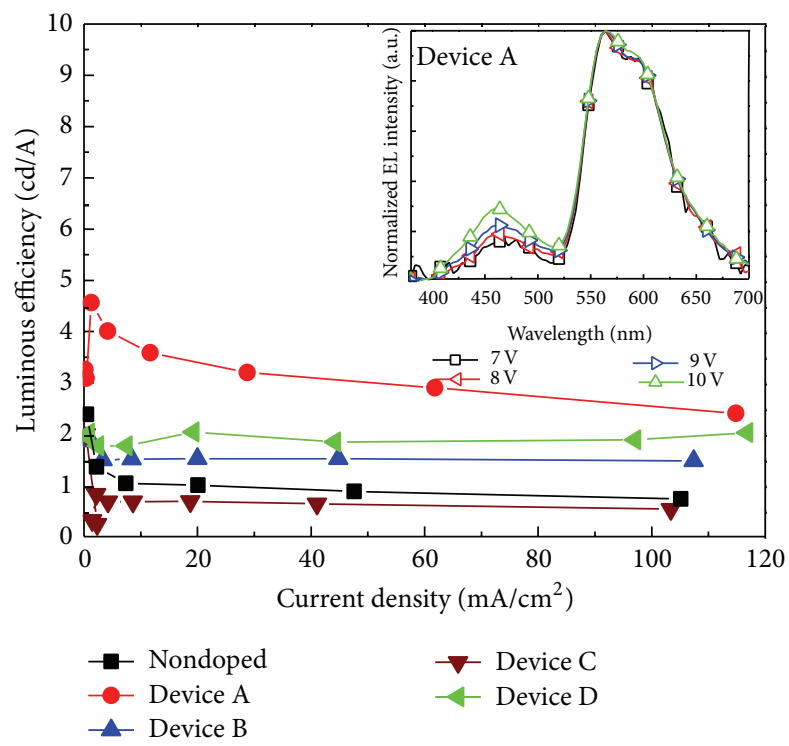

(c)

FIGURE 5: The (a) current density-voltage, (b) luminance-voltage, and (c) luminous efficiency-current density characteristics of the various structures. The inset shows the normalized EL intensity spectra of the device A at the applied voltage of 7-10 V.

indicates that stable luminous efficiency can be achieved by the improved carrier balance (as confining of holes).

\section{Conclusion}

In summary, the optimized structure of ITO/ $\mathrm{MoO}_{3}(5 \mathrm{~nm}) /$ NPB $(35 \mathrm{~nm}) / \mathrm{CBP}$ (HBL1) $(5 \mathrm{~nm}) / \mathrm{DPVBi}$ (I) $(10 \mathrm{~nm}) / \mathrm{CBP}$ (HBL2): Rubrene $(4: 1)(3 \mathrm{~nm}) / \mathrm{DPVBi}$ (II) $(30 \mathrm{~nm}) / \mathrm{CBP}$ (HBL3) $(2 \mathrm{~nm}) / \mathrm{BPhen}(10 \mathrm{~nm}) / \mathrm{LiF} / \mathrm{Al}$ which generate white emission was fabricated. The result showed that the device with Rubrene doped in CBP (HBL2) exhibited a stable white emission with the color coordinates of $(0.322,0.368)$ and coordinates slight shift of $\pm \Delta_{x, y}=(0.001,0.011)$ at applied voltage of $8-12 \mathrm{~V}$. When the thicknesses of DPVBi and CBP layer have too much or less, the properties of WOLED will be variable. Thus, the thicknesses of DPVBi and CBP layer must be properly controlled to achieve the stable color purity and high efficiency of WOLED in THBL.

\section{Conflict of Interests}

The authors declare that there is no conflict of interests regarding the publication of this paper. 


\section{Acknowledgment}

This work was partially supported by the National Science Council of the Republic of China under Contract no. NSC 102-2221-E-390-019-MY2.

\section{References}

[1] C. W. Tang and S. A. Vanslyke, "Organic electroluminescent diodes," Applied Physics Letters, vol. 51, no. 12, pp. 913-915, 1987.

[2] R. H. Friend, R. W. Gymer, A. B. Holmes et al., "Electroluminescence in conjugated polymers," Nature, vol. 397, no. 6715, pp. 121-128, 1999.

[3] H. Aziz, Z. D. Popovic, N. X. Hu, A. M. Hor, and G. Xu, "Degradation mechanism of small molecule-based organic light-emitting devices," Science, vol. 283, no. 5409, pp. 19001902, 1999.

[4] Y. Wang, Y.-L. Hua, X.-M. Wu, L.-J. Zhang, Q.-C. Hou, and Q. Liu, "High-efficiency fluorescent white organic light-emitting device with double emissive layers," Organic Electronics, vol. 9, no. 2, pp. 273-278, 2008.

[5] P. Chen, W. Xie, J. Li et al., "White organic light-emitting devices with a bipolar transport layer between blue fluorescent and orange phosphorescent emitting layers," Applied Physics Letters, vol. 91, no. 2, Article ID 023505, 3 pages, 2007.

[6] M.-Y. Chang, C.-H. Wang, S.-C. Lin, and Y.-F. Chen, "Highbrightness, high-color-purity, white organic light-emitting diodes featuring multiple emission layers," Journal of Applied Physics, vol. 105, no. 6, Article ID 064318, 6 pages, 2009.

[7] C.-H. Hsiao and J.-H. Lee, "Emitting-layer design of white organic light-emitting devices with single-host material," Journal of Applied Physics, vol. 106, no. 2, Article ID 024503, 7 pages, 2009.

[8] H. Yang, Y. Shi, Y. Zhao et al., "High colour rendering index white organic light-emitting devices with three emitting layers," Displays, vol. 29, no. 4, pp. 327-332, 2008.

[9] S.-H. Yang, B.-C. Hong, and S.-F. Huang, "Luminescence enhancement and emission color adjustment of white organic light-emitting diodes with quantum-well-like structures," Journal of Applied Physics, vol. 105, no. 11, Article ID 113105, 7 pages, 2009.

[10] H.-P. Lin, D.-B. Yu, X.-W. Zhang et al., "Enhancing color purity and efficiency of white organic light-emitting diodes using a double-emitting layer," Semiconductor Science and Technology, vol. 25, no. 10, Article ID 105004, 2010.

[11] S.-S. Lee, D. Ko, C.-H. Chung, and S. M. Cho, "Movement of carrier recombination zone in organic light emitting devices by applied voltage," Synthetic Metals, vol. 128, no. 1, pp. 51-55, 2002.

[12] X. Y. Zheng, W. Q. Zhu, Y. Z. Wu et al., "A white OLED based on DPVBi blue light emitting host and DCJTB red dopant," Displays, vol. 24, no. 3, pp. 121-124, 2003.

[13] J.-H. Jou, Y.-S. Chiu, R.-Y. Wang, H.-C. Hu, C.-P. Wang, and H.-W. Lin, "Efficient, color-stable fluorescent white organic light-emitting diodes with an effective exciton-confining device architecture," Organic Electronics, vol. 7, no. 1, pp. 8-15, 2006.

[14] Y.-S. Seo and D.-G. Moon, "Highly efficient blue phosphorescent organic light-emitting devices using simple structures with thin 1,1-bis[(di-4-tolyamino)phenyl]cyclohexane layers," Synthetic Metals, vol. 160, no. 1-2, pp. 113-115, 2010.

[15] J. Zhu, W. Li, B. Chu et al., "Non-doped-type white organic light-emitting diodes for lighting purpose," Journal of Luminescence, vol. 130, no. 5, pp. 865-868, 2010.
[16] S.-H. Yang and D.-W. Zhuang, "Enhancement of efficiency of multilayer polymer light-emitting diodes by inserting blocking layers," Journal of Luminescence, vol. 131, no. 4, pp. 801-807, 2011.

[17] Q. Xue, S. Zhang, G. Xie et al., "Efficient fluorescent white organic light-emitting devices based on a ultrathin 5,6,11,12tetraphenylnaphthacene layer," Solid-State Electronics, vol. 57, no. 1, pp. 35-38, 2011.

[18] H. Mattoussi, H. Murata, C. D. Merritt, Y. Iizumi, J. Kido, and Z. H. Kafafi, "Photoluminescence quantum yield of pure and molecularly doped organic solid films," Journal of Applied Physics, vol. 86, no. 5, pp. 2642-2649, 1999.

[19] Y. Wang, F. Teng, Z. Xu, Y. Hou, S. Yang, and X. Xu, “Trap effect of an ultrathin DCJTB layer in organic light-emitting diodes," Materials Chemistry and Physics, vol. 92, no. 2-3, pp. 291-294, 2005.

[20] M. Matsumura and T. Furukawa, "Efficient electroluminescence from a rubrene sub-monolayer inserted between electron- and hole-transport layers," Japanese Journal of Applied Physics A, vol. 40, no. 5, pp. 3211-3214, 2001.

[21] T. Li, X. Li, W. Li et al., "Tunable red emission by incorporation of a rubrene derivative in $p$-type and $n$-type hosts in organic light emitting devices," Thin Solid Films, vol. 517, no. 16, pp. 4629-4632, 2009.

[22] Q. Xue, G. Xie, P. Chen et al., "White organic light-emitting devices with a bipolar transport layer between blue fluorescent and yellow phosphor-sensitized-fluorescent emitting layers," Synthetic Metals, vol. 160, no. 7-8, pp. 829-831, 2010.

[23] J. Zhao, J. Yu, L. Zhang, and J. Wang, "Non-doped phosphorescent white organic light-emitting devices with a quadruplequantum-well structure," Physica B: Condensed Matter, vol. 407, no. 14, pp. 2753-2757, 2012.

[24] C.-J. Huang, T.-H. Meen, K.-C. Liao, and Y.-K. Su, "The mechanism of efficiency enhancement with proper thickness of DPVBi layer for blue organic light-emitting devices (BOLED)," Journal of Physics and Chemistry of Solids, vol. 70, no. 3-4, pp. 765-768, 2009.

[25] H.-P. Lin, F. Zhou, J. Li et al., "A high performance of BPhenbased white organic light-emitting devices with a dual-emitting layer and its electroluminescent spectral property," Journal of Industrial and Engineering Chemistry, vol. 17, no. 4, pp. 675-680, 2011.

[26] L. Wen, F. Li, J. Xie et al., "Electroplex emission at PVK/Bphen interface for application in white organic light-emitting diodes," Journal of Luminescence, vol. 131, no. 11, pp. 2252-2254, 2011.

[27] B. Ding, W. Zhu, X. Jiang, and Z. Zhang, "White organic light-emitting diodes based on incomplete energy transfer from perylene to rubrene," Solid State Communications, vol. 148, no. 5-6, pp. 226-229, 2008.

[28] C. J. Huang, T. H. Meen, S. L. Wu, and C. C. Kang, "Improvement of color purity and electrical characteristics by co-doping method for flexible red-light organic light emitting devices," Displays, vol. 30, no. 4-5, pp. 164-169, 2009. 

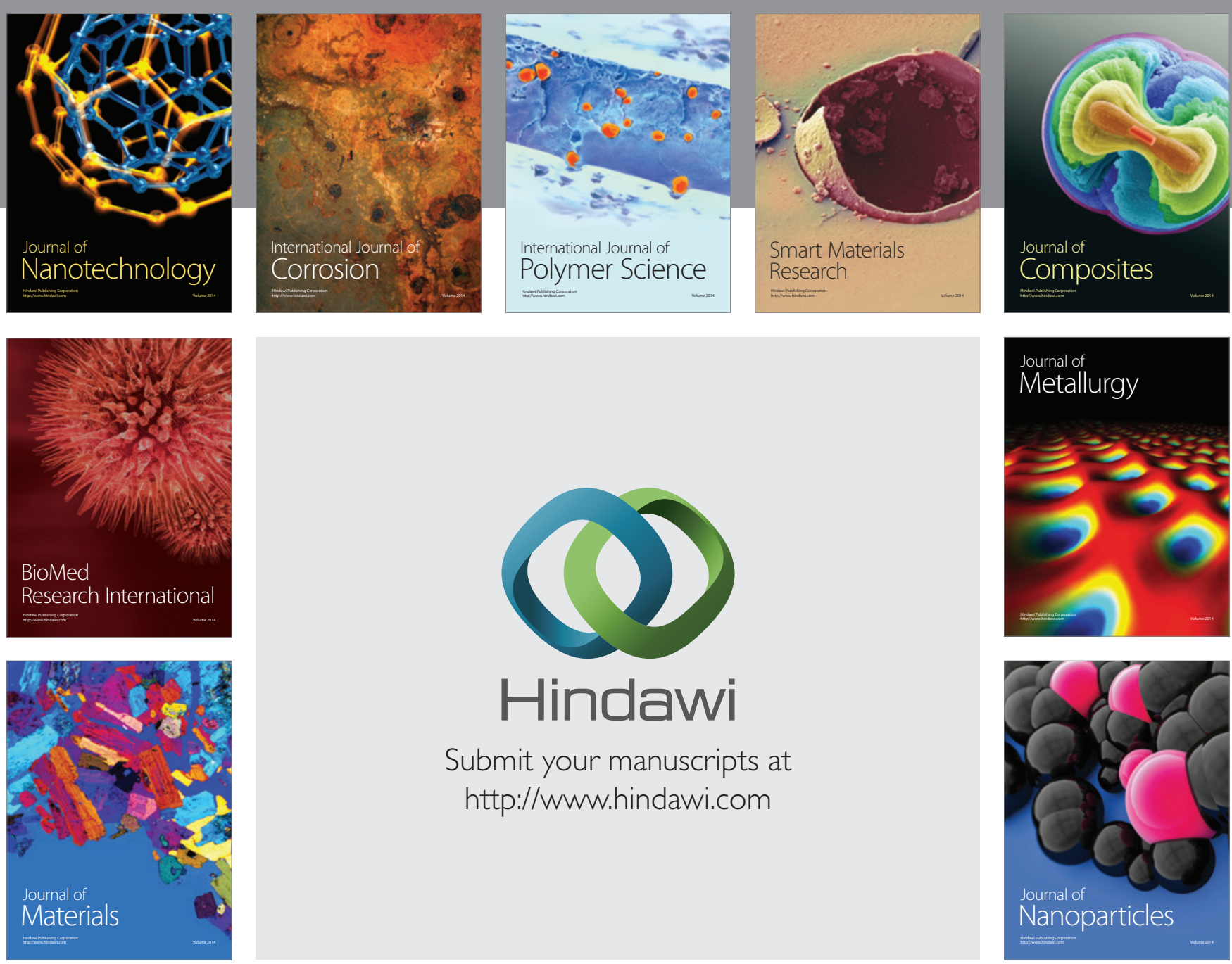

Submit your manuscripts at http://www.hindawi.com
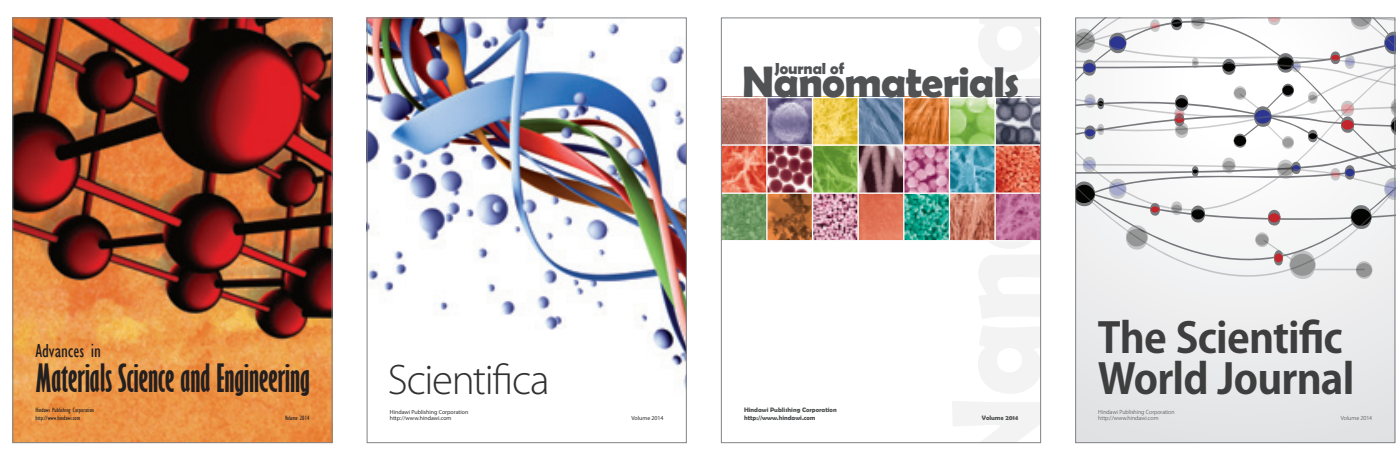

\section{The Scientific World Journal}
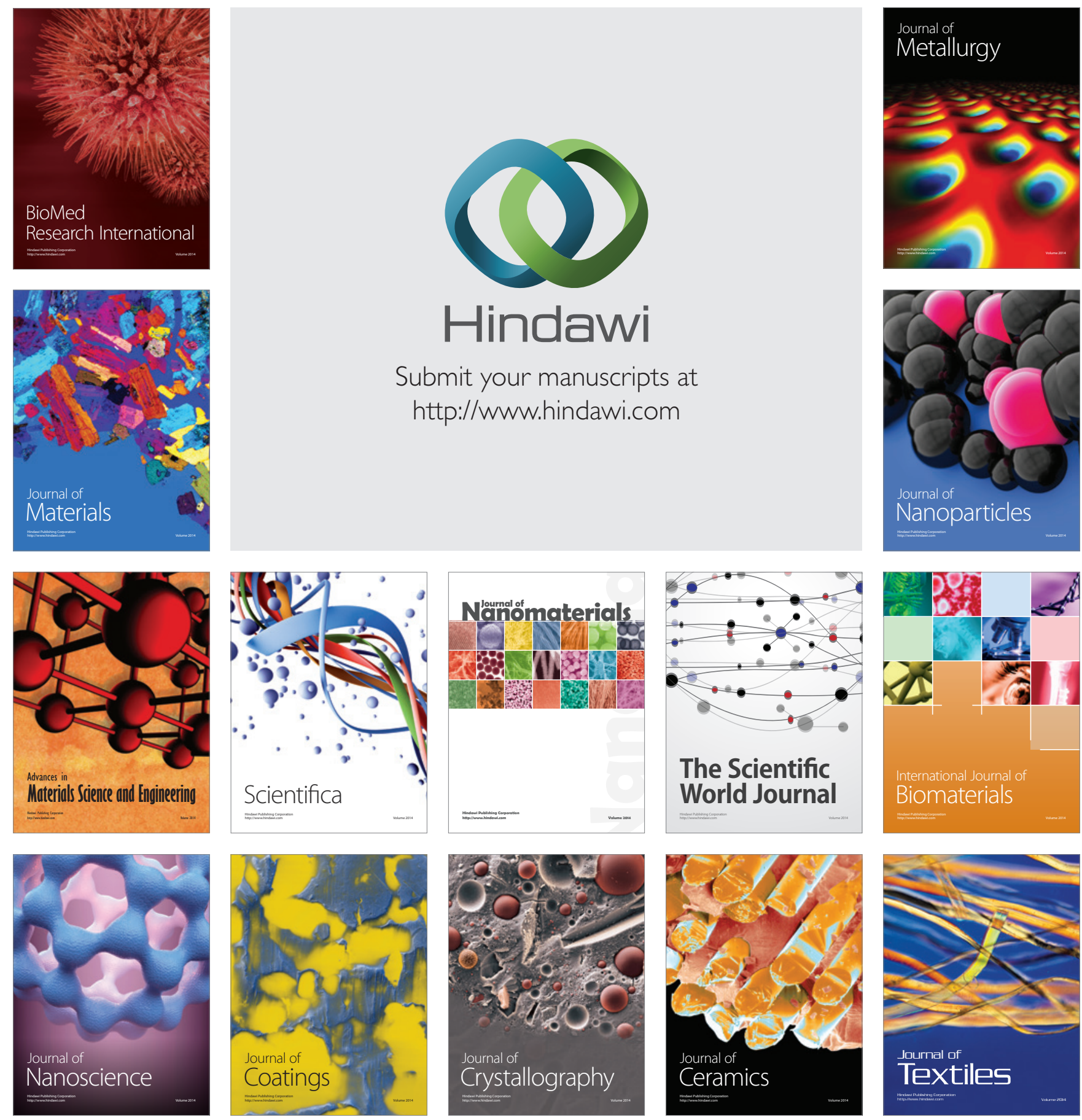